\title{
Where to Start with AI? - Identifying and Prioritizing Use Cases for Health Insurance
}

\author{
Michael Weber \\ Technical University of Munich \\ mic.weber@tum.de
}

\author{
Nicolas Limmer \\ PricewaterhouseCoopers $\mathrm{GmbH}$ \\ nlimmer@outlook.com
}

\author{
Jörg Weking \\ Technical University of Munich \\ joerg.weking@tum.de
}

\begin{abstract}
Artificial Intelligence (AI) arguably represents a key technology for the digitalization of health care. Specifically, health insurers can benefit from AI as they typically have access to vast amounts of data. However, practitioners struggle to adopt AI in productive use, and extant research lacks an overview of use cases for AI in health insurance as well as prioritization criteria that can guide their implementation. To address this gap, we conduct explorative interviews in the context of the German statutory health insurance system. We identify AI use cases in the areas of predictive health, individualized service, anomaly detection, and operations enhancement. We find that health insurers are likely to prioritize these use cases according to implementation complexity and business orientation, whereas focusing on simple use cases that target cost savings is recommended by experts. Our study advances the understanding of AI adoption in health insurance and supports practitioners in guiding future AI initiatives.
\end{abstract}

\section{Introduction}

Artificial Intelligence (AI) is widely viewed as a key technology for the digitalization of health care [1]. AI compromises a broad suite of different technologies, including machine learning, computer vision, natural language processing, and robotics [2]. It can be applied in health care to augment diagnosis generation and therapy selection, predict risks and diseases, reduce medical error, or enhance productivity [1, 3]. For example, computer vision can be leveraged to augment doctors' capabilities in cancer detection [4], or augmented reality can support wound management for hands-free service innovation [5]. By improving quality and efficiency in health care, AI is expected to lower health expenditures by billions within the next 10 years, even when only considering Europe [6].

While all stakeholders are expected to benefit from AI, health insurers, especially, are in a promising position as they represent the central interface among service providers, producers, and insured persons. For them, new opportunities come through using the vast amounts of data they possess, especially health-related data [7]. These data enable the broad use of AI across all business areas in health insurance. Furthermore, regulatory adjustments allow the data to be further used effectively in a way that was not possible before. By using these data to implement AI systems, health insurers are expected to achieve internal improvements through cost savings and process optimization, including increased customer satisfaction through improved quality of care and individualized communication with insured persons.

Nevertheless, AI adoption proves to be a challenging endeavor in practice [8, 9]. Healthcare organizations, especially, are facing multiple challenges for AI adoption, including strong regulations, the sensitivity of health data, the integration of AI systems into existing workflows, patient safety and acceptance, and the need for ethical considerations [3, 10]. Given these challenges and the high failure rate of AI projects in practice [8], organizations need to carefully prioritize and select use cases for AI adoption [11].

Against this background, current research on AI adoption faces two limitations. First, despite health insurers' promising position, current research still lacks an in-depth investigation of AI adoption for health insurance. Findings from other domains might not transfer seamlessly [e.g., 12, 13, 14], as they do not account for context-specific aspects. Second, extant research on AI adoption predominately views $\mathrm{AI}$ as a monolithic concept, meaning that $\mathrm{AI}$ is viewed as a homogeneous set of use cases and technologies. However, AI can be applied in a plethora of use cases while using different technologies [2]. Hence, a more nuanced perspective on different use cases should be applied in researching AI adoption to develop a better understanding of how AI use cases are prioritized in practice. More research is needed in these areas to provide guidance for AI adoption in health insurance. 
To address this, we ask the following research questions:

\section{1) What are use cases for AI in health insurance?}

\section{2) How do health insurers prioritize AI use cases?}

To answer these research questions, we conducted explorative interviews with experts from industry and consulting in the German statutory health insurance system. This approach allowed us to explore AI use cases in health insurance and derive criteria that are applied for use case prioritization. We identified a plethora of AI use cases in the areas of predictive health, individualized service, anomaly detection, and operations enhancement. Furthermore, we discovered that health insurers are likely to prioritize AI use cases according to implementation complexity and business orientation. Experts recommend health insurers with little experience in AI and digital technologies to start with simple use cases that primarily target cost savings and efficiency gains. More complex use cases typically require more investment and, in some instances, change current service portfolios. This observation also highlights the importance of strategic considerations for AI use case prioritization.

This study contributes to the discourse on AI adoption within and outside health care. We enhance our understanding of AI adoption in health insurance with its unique regulatory context and the sensitivity of the data. We provide a novel perspective on AI adoption by differentiating between use cases (with distinct characteristics), instead of viewing AI as monolithic concept; research and practice should consider AI adoption in a more nuanced way. Health insurance practitioners might use our findings as a starting point for their AI adoption decision making. We provide a structured list of AI use cases and criteria for use case prioritization.

\section{Background}

For the background of this study, we briefly describe the context of the German statutory health insurance system. Thereafter, we present related work on AI adoption within and outside health care.

\subsection{German Statutory Health Insurance}

Statutory health insurance is part of the German social system and central to their healthcare system. It is meant to "maintain, restore, or improve the health of the insured" ( $\$ 1$ German Social Code V). Alongside private health insurers, they are the main funding bodies for the services provided in the German healthcare system. The future viability of the healthcare system has increasingly become the focus of political and public attention in recent years. Statutory health insurers are caught between a shortage of skilled workers, funding gaps, and a nursing shortage against digitization, innovation, and new technologies [15]. On the one hand, statutory health insurers are required to reduce expenses for service and administrative tasks; on the other hand, they inevitably need to undergo costly digital transformation to remain competitive [16]. The effective use of the data that health insurers have at their disposal is expected to play a central role in this scenario [7]. Making use of their data can be achieved with the help of AI, but health insurers need to adequately prepare for this strategically.

\subsection{Related Work on AI Adoption}

Extant research has identified a plethora of factors that influence the AI adoption decision-making process. Various contexts have been studied, including public health care, car manufacturing, tourism, and financial industries [10, 12-14, 17]. Following the works of Depietro, et al. [18] and Rogers [19], these influencing factors can be organized using the technology, organization, and environment framework (Figure 1).

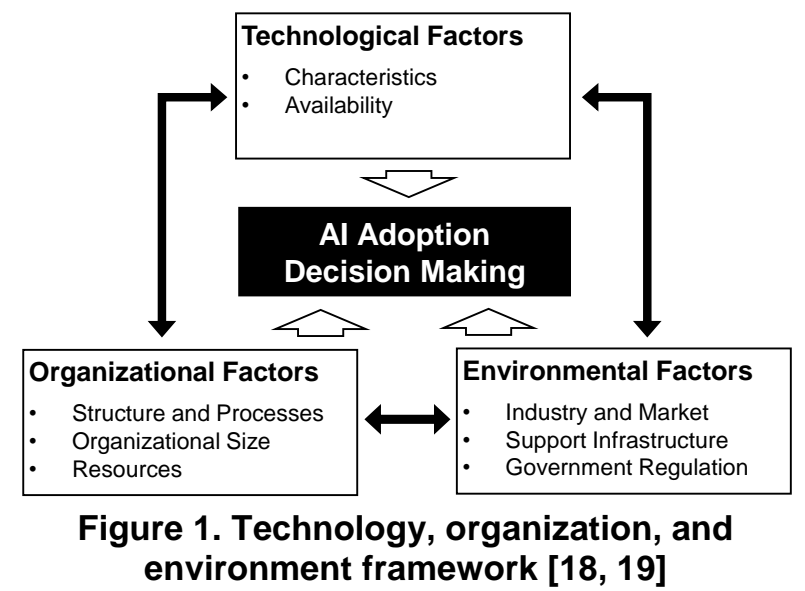

From a technological perspective, several factors influence AI adoption. First, organizations need to assess whether AI technology provides a relative advantage as compared with traditional IT [13]. AI implementation typically is more complicated, which might raise concerns about its benefits. Second, organizations require the presence of adequate IT infrastructure for AI, which compromises AI-specific tools (e.g., AI engines and analytical sandboxes) as well as the compatibility of existing IT systems with AI systems $[9,17,20]$. Third, organizations require training data in sufficient quality and quantity for AI development $[12,21]$. This requirement is especially 
relevant because today's AI systems are mostly based on machine learning from data.

From an organizational perspective, AI adoption is facilitated by the presence of several factors. First, organizations need access to specific human resources and expertise to implement AI $[9,22]$. Beyond technical know-how, this access to human resources and expertise also includes AI awareness in management and "AI translators" [22]. Second, research has highlighted the need for top management commitment as AI adoption typically requires larger investments $[12,14,22]$ where a dedicated AI budget is expected to drive AI adoption in organizations [12]. Third, employee acceptance has been raised as an important issue, because they can fear replacement through AI $[13,17]$. Therefore, an open, experimental, and data-driven culture is expected to drive AI adoption [23].

From an environmental perspective, different external factors impact organizational AI adoption. First, this includes government regulations and industry specifics [24]. For example, regulations like the General Data Protection Regulation (GDPR) complicate AI adoption in most industries. Moreover, certain regulated industries like public health care or car manufacturing tend to face additional challenges [10, 13]. Second, a competitive market might drive AI adoption [17]. For example, financial services industries are facing fintech startups as competitors that, in some instances, very successfully rely on AI systems [14]. Novel startups and business models can also be observed in health care [cf. 25]. Third, customer AI readiness has been mentioned as an important factor [17, 22]; a customer group might not want to communicate with an AI system for various reasons [17], such as when the system produces wrong or discriminating outputs.

Current research faces two limitations when regarding AI adoption in health insurance. First, only few studies have considered AI adoption in healthcare insurance, despite the great potential and particular challenges for AI adoption in this domain [10]. Second, many factors have been identified across industries that influence the adoption of AI technology, but our understanding of how organizations go about selecting and prioritizing different use cases to drive AI adoption remains limited. Hence, we explore AI use cases and prioritization criteria for health insurance.

\section{Research Approach}

We selected an interpretive research stance [26] to explore AI use cases and possible prioritization criteria for health insurance, as AI adoption is still at an early stage. We conducted exploratory interviews with health insurance experts, including managers, developers, data scientists, and consultants, allowing the representation of many different voices and viewpoints [27] on AI adoption. Nevertheless, we had to keep in mind that the interviewees only reported on their personal viewpoints and experiences [28]. In the following, we depict our approach to data collection and data analysis.

\subsection{Data Collection}

For data collection, we conducted exploratory interviews with health insurance experts. We prioritized experts in different roles and positions with AI experience, including managers, developers, data scientists, and consultants. By including both experts from industry and consulting (pseudonymized as IN and $\mathrm{CO}$, respectively), we were able to cover internal and external perspectives. This sampling allowed us to capture many different viewpoints, which in turn helps addressing potential biases in the data [29]. Although we managed to interview both technical and business experts within the external group of interviewees, we could not interview an individual holding a business role within the internal group. We were declined multiple times, as the COVID-19 pandemic kept health insurers busy during our study. Table 1 provides an overview of the interviews.

Table 1. Interview partners

\begin{tabular}{|l|l|r|r|}
\hline ID & Position & $\begin{array}{l}\text { Duration } \\
\text { (min) }\end{array}$ & Date \\
\hline IN1 & Data Scientist & 51 & $11 / 2020$ \\
\hline IN2 & Senior Data Scientist & 31 & $12 / 2020$ \\
\hline IN3 & Software Developer & 34 & $12 / 2020$ \\
\hline CO1 & Senior Consultant & 39 & $10 / 2020$ \\
\hline CO2 & Director & 23 & $10 / 2020$ \\
\hline CO3 & Manager & 37 & $10 / 2020$ \\
\hline CO4 & Senior Consultant & 28 & $11 / 2020$ \\
\hline
\end{tabular}

We conducted a total of seven interviews between October and December 2020. We used semi-structured interview guidelines, which allowed for a certain level of flexibility to explore interesting directions [27]. We asked our experts on existing and planned AI initiatives within companies, other potential use cases, and criteria that guide use case prioritization along the AI adoption journey. The interviews lasted $35 \mathrm{~min}$ on average. Some interviewees provided brief responses, while others provided rich information even beyond the scope of this study. We recorded and transcribed each interview for data analysis and interpretation. 


\section{1st-Order Concepts}

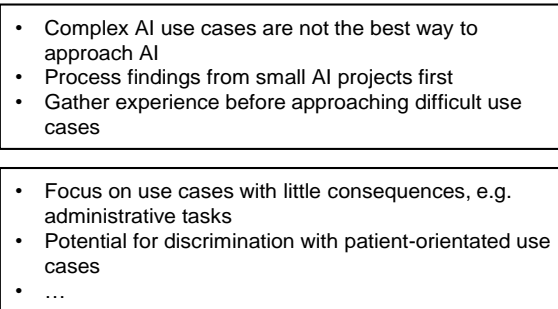

- Easier to start with rich available data, e.g. data on invoices or diagnoses

- Legal obstacles when reaching out for data, e.g. for hospitals, pharmacies, physicians

\section{2nd-Order Themes Aggregate Dimensions}

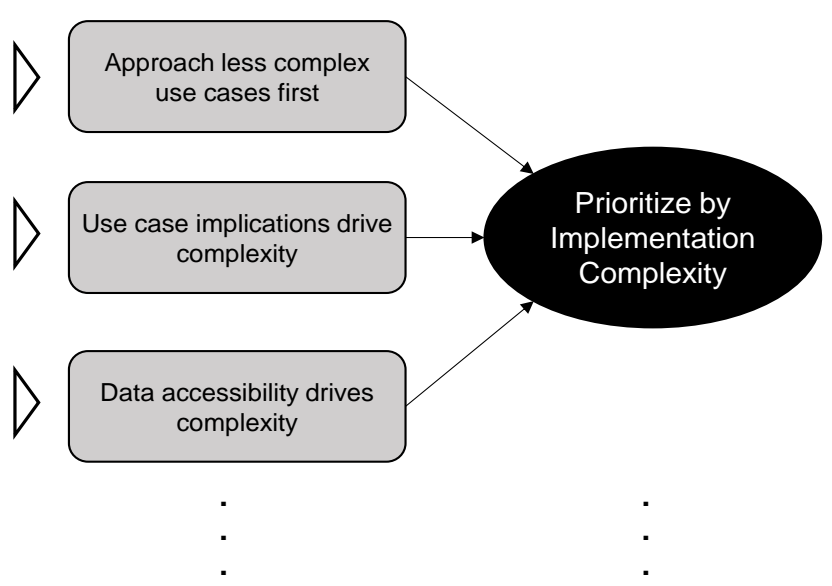

Figure 2. Exemplary Coding scheme

\subsection{Data Analysis}

For data analysis, we followed an iterative approach that included data coding with increasing levels of abstraction as well as the constant comparison of the data with our emergent findings [29]. After five to six interviews, we did not gain substantially new insights for the purpose of our explorative study. Hence, data collection stopped after seven interviews and data analysis began. The analysis was guided by our understanding of the topic, which we built from prior literature (cf. Section 2.2) and our own fieldwork. While one researcher took the lead in data coding, we regularly discussed the emergent findings within the research team and checked for potential biases.

We coded our data following the procedure by Gioia, et al. [30], as we sought to find similarities and differences in the data. As a first step, we coded our data with first-order codes, which were closely aligned to the domain and language of the interviewed experts. We then organized and grouped them into second-order themes, which are closer to the researchers' domain. Thereafter, we derived theoretical aggregate dimensions from the second-order themes. This last step allowed us to organize and interpret our findings. Figure 2 illustrates the coding scheme.

\section{Results}

We identified a plethora of AI use cases in the areas of predictive health, individualized service, anomaly detection, and operations enhancement. Our data led us to suggest that health insurers prioritize these use cases according to implementation complexity and business orientation. In the following, we will depict these findings in more detail.

\subsection{AI Use Cases for Health Insurance}

We identified a plethora of use cases for AI, and as a result of our coding procedure, we grouped these use cases into four categories: predictive health, individualized service, anomaly detection, and operations enhancement (Table 2).

First, health insurers can apply AI for predictive health and service management. Here data from doctors, hospitals, insured persons, and drug producers can be integrated for promising use cases for predictive and preventive health care. Examples include the early detection of various diseases, the management of disease progressions, or the prediction of potential drug interactions. For instance, personal movement data might be leveraged to predict bouts of depression, potentially preventing diseases or enhancing current treatment and curing procedures. This also holds great potential for health insurers to save costs: "an insured person who is healthy also incurs fewer costs with a health insurance company" (IN2). This makes predictive health and service management especially attractive for stationary care, which is a key cost driver for health insurers: "If you look at the annual reports and also the costs of the health insurance funds, then you see that hospitals are the biggest cost drivers. There is an extremely large savings potential there" (CO1).

Second, health insurers can apply AI for individualized service and communication. Using the AI models and individual data of the insured, health insurers can offer individualized care services and analyze customer interaction data to try predicting and preventing customer churn. Although individualized services and communication are not new, our data suggest that AI allows us to carry out this task more efficiently and effectively, as senior data scientist IN2 
describes: "You can imagine that such initiatives already exist on a large scale among statutory health insurers. In other words, they approach the insured and make them offers on how their health can be improved. Until now, however, this has been relatively unstructured and untargeted. And because we now have data going back many years, we can simply unleash a machine learning algorithm on it and see whether this algorithm uncovers patterns that a human worker may not have been aware of before" (IN2).

Third, health insurers can apply AI for anomaly detection. Fraud detection for insurance claims represents a promising use case here, especially to save costs. Our data suggest that some health insurers have already implemented this. From their perspective, "it was clear very early on that this is a big issue. That's why it was one of the first projects that was tackled, and accordingly it is already in a productive environment" (IN2). Additionally, following a new German regulation from 2020, "a health insurance company has now only the possibility to give a certain percentage of invoices [to an external auditor ...]" (CO1), which makes this use case even more attractive. Another high potential area is the detection of incorrect medications, as this typically means "that a patient is now receiving too much or the wrong medication and therefore needs something else. This makes the whole case more expensive overall" (CO3).

Fourth, health insurers can apply AI for operations enhancement. The main purpose is to automate and streamline supporting processes within the company, including insurance claims processing, payment order verification, or customer support. For instance, health insurers might apply AI to automatically process followup prescriptions, "so that there's not a person having to sift through a prescription again and again, which just leads to a lot of fairly trivial decisions being taken away and [we] speed up that whole process a little bit" (IN2). Another example, chatbots might be integrated into customer support to automate customer service and consultation (IN3). Implementing such might "lower the administrative overhead, leading to faster response times. This also leads to greater satisfaction on the part of the insured and more money for other things" (IN1). Nevertheless, the interviewees propose that the business value potential for operations enhancement is lower as compared with that of predictive health and anomaly detection.
Table 2. Al Use Cases for Health Insurance

Predictive Health and Service Management

- Prediction and early detection of diseases, e.g., strokes, heart attacks, or diabetes

- $\quad$ Prediction and management of disease progressions

- Prediction of preventive measures against diseases

- Prediction of potential drug interactions

- Prediction of customer costs for service management

\section{Individualized Service and Communication}

- Generation of individualized services and tariffs

- Analyses of customer interaction data for customer communication management

- Prediction of customer churn

- Independent consultation through an AI bot

Anomaly Detection

- Fraud detection for insurance claims

- Detection of incorrect medications

\section{Operations Enhancement}

- Automation of internal document processing

- Automated verification of insurance applications or payment orders

- Management and steering of internal processes

- Automation of customer support through chatbots

\subsection{Prioritization Criteria for AI Use Cases}

Beyond identifying AI use cases, we further asked our experts how they prioritize these use cases as part of their AI adoption. We discovered two categories of criteria that likely influence prioritization: implementation complexity and business orientation. Following these criteria, health insurers are likely to prioritize different use cases for actual implementation. Table 3 provides an overview of the identified criteria. In the following, we depict our findings in more detail.

\section{Table 3. Prioritization criteria for Al use cases}

\begin{tabular}{|l|ll|}
\hline \multicolumn{1}{|c|}{ Aggregate dimension } & \multicolumn{2}{|l|}{ Use case criteria } \\
\hline Implementation & $\bullet$ & Use case implications \\
complexity & $\bullet$ & Data accessibility \\
& $\bullet$ & Data sensitivity \\
\hline Business orientation & $\bullet$ & Use case value \\
& $\bullet$ & Strategic alignment \\
\hline
\end{tabular}


4.2.1 Implementation Complexity. Our data suggest that health insurers prioritize according to implementation complexity, which we view as a technological factor. There are manifold AI use cases for health insurance, and as AI was a novel topic for the health insurers in our sample, the common theme is to start with simple use cases (CO2). This would allow health insurers "to process findings from the first projects correctly and also to draw the right conclusions from them in order to be able to scale [...] and to be able to realize the added values of AI across the entire organization" (CO1). For our interviewed experts, this observation would mean to focus on operations enhancement and anomaly detection first (CO2). The "more complex [use] cases in the area of prediction of disease progression and [...] also individualized customer service, [are] perhaps not the best solution in my opinion to approach the topic of AI" (CO1). More complex use cases should then only be approached by more experienced organizations. Figure 3 illustrates the possible prioritization of AI use cases according to implementation complexity for German statutory health insurers. Figure 3 classifies use cases based on our qualitative data. It indicates the interviewees' individual assessments and thoughts, rather than ground truth facts or a quantitative ranking.

Our data suggest that the implementation complexity of AI use cases can be described by three criteria. First, use case implications influence implementation complexity. Here, use cases that are solely working internally (and not facing the customer) tend to be simpler to realize. As a health insurance employee states, "the things with little consequences are processed first -that is, for the insured. So, you first automate the administration, which of course already leads to benefits" (IN1). In other use cases, such as predictive health or individualized services, the AI models' decisions can have serious consequences (CO3). A health insurance data scientist explains: "since it is a statistical procedure, [...] you have to make sure that no patients are excluded from a program that might be useful for them and that they are not put in a worse position [...] This must not be the case" (IN1). Here, the decision of the AI system typically needs to be reasonable and explainable to customers. This helps to address potential issues of discrimination (IN2).

Second, data accessibility needs to be considered for implementation complexity. Health insurers typically have a large pool of high-quality data that can and should be used early on (e.g., data on diagnoses or invoices (CO4)). As a consulting director reported, "statutory health insurers have data in use cases [such as] service management, drugs, drug fraud, and so on, and there are opportunities to quickly develop best practices with existing data and very inward-looking use cases" (CO2). If the data required for a use case are not easily accessible, this would imply cooperating with other parties, like other health insurers or hospitals. This typically leads to additional complexity and potential barriers for use case implementation. A health insurance

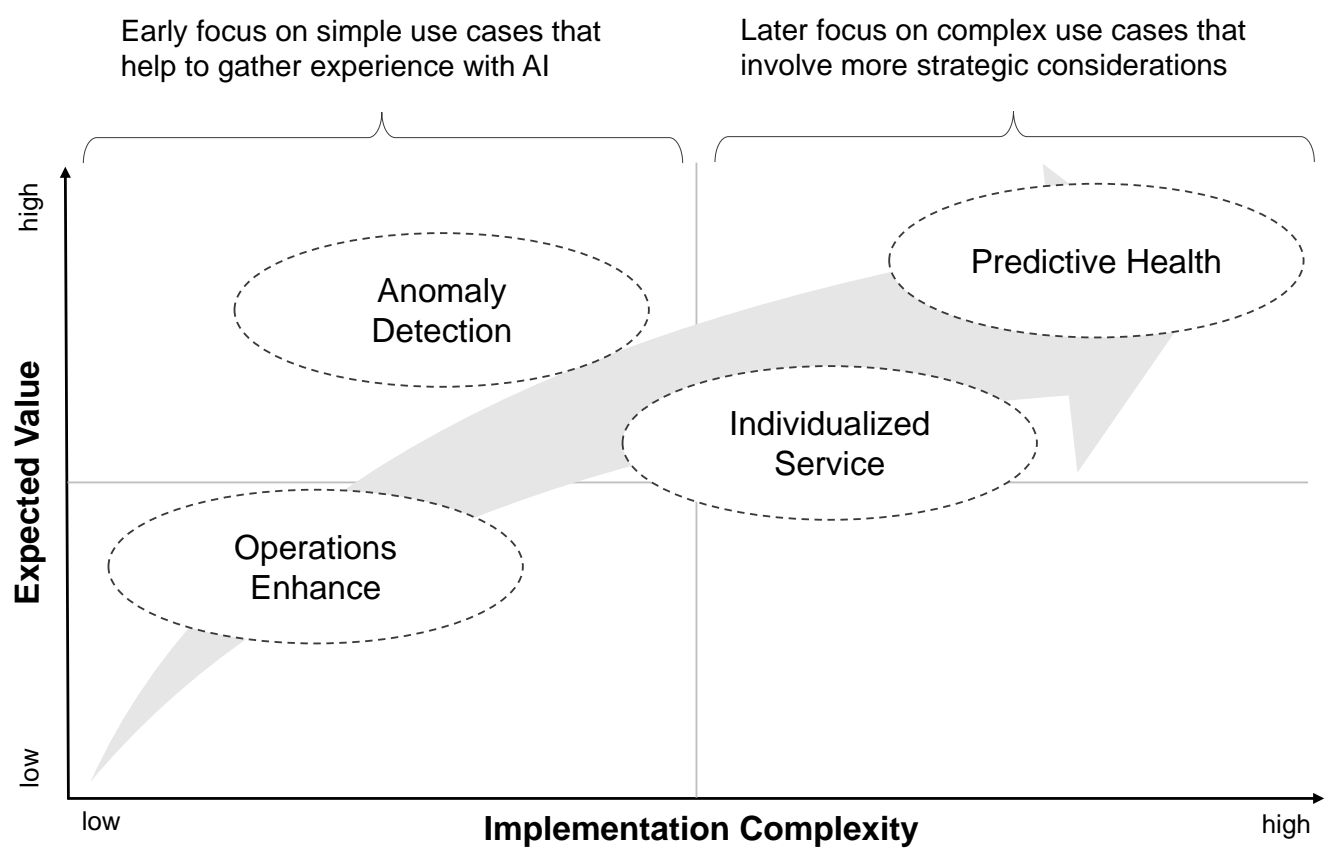

Figure 3. Al Use Case Prioritization by Implementation Complexity

Note: The Al use cases have been classified qualitatively. The figure indicates the interviewees' individual assessments and thoughts, rather than ground truth facts. 
employee explains, it is even "outside of [their] current legal ability to actively reach out to hospitals, physicians, pharmacies, and so forth and ask them to share data with us so that we can improve our analytics" (IN3).

Third, the data sensitivity required for the use case also plays an important role as there is often sensitive and personal data involved that need to be protected at all costs, which adds to implementation complexity. Having a data leak "would really be the worst-case scenario, because you can't have any more sensitive data than that what we have as data" (IN3). To address this, dedicated security mechanisms need to be implemented (IN2). Health insurers need to adhere to strong German regulations (e.g., the GDPR or the social code $(\mathrm{CO} 3))$. This also implies that health insurers cannot use all data for all purposes. Instead, they need to "have as legal a mandate to do that" (IN3). Here, working with less sensitive and regulated data is simpler to start with at the beginning of the AI adoption.

4.2.2 Business Orientation. Besides implementation complexity, our data suggest that health insurers should also prioritize use cases according to their business orientation, which we view a predominately organizational factor. Other than in the case of implementation complexity (focus on simple use cases first), our interviewed experts did not have a clear recommendation here. Instead, a health insurer needs to assess its own business orientation and prioritize AI use cases accordingly to fit its needs.

One criterion to consider is the expected use case value for business. The previously identified categories of use cases focus on different value targets. As an example, predictive health addresses the general prevention of diseases, which is expected to reduce harm and insurance costs (CO3). As a senior data scientist explains, if "statutory health insurers raise the health of their insureds so much [...] you will of course also be able to press the costs there" (IN2). As another example, operations enhancement is expected to optimize back-office processes, which drives internal efficiency. "Where an application used to take three weeks to process, it now could be done in a matter of hours, if not minutes" (IN3). This saves resources for health insurers and might also lead to more satisfied customers (IN2). Although all value targets are highly relevant for health insurers, the current situation around the COVID-19 pandemic led some experts to argue that cost savings should be prioritized early on. As a senior consultant puts it, "especially with the pandemic situation and also with the financial holes that are in the budget of the health insurance companies, of course the focus one and the focus two and the focus three is on cost savings" (CO1). This mostly complements the focus on internal and less complex use cases (cf. Figure 3 ), as "cost savings is something which can be quickly achieved" (CO2).

Besides use case value, the strategic alignment of health insurers with use case implementation is an important criterion. A consulting manager explains, "it's a strategic decision. It depends a little bit on whether you think you have to become digital organization really, really fast and you also invest what it takes or whether you say, 'I want [...] to try it first and see how it is and see how it develops" (CO3). Some use cases require larger investments or other strategic decisions for successful implementation. For example, to gather enough data to train an AI model, collaborations and sharing of data with external partners might be required. "When several health insurance companies join forces, the data pool is of course better and the quality of an AI model is better" (IN2). However, from competitors' perspective, that is potentially undesirable, especially if the health insurer already has a large data pool (IN2). In that case, it is a strategic decision for a health insurer to prioritize a use case that starts collaborations with external partners. As another example, certain use cases go beyond incremental improvements and have strategic implications for the business model. Like with predictive health, these use cases would typically augment the existing service portfolio. It would enable a health insurer to "position itself as a health advisor with the data it has on the insured and go more into prevention instead of [...] the previous role, which is more in benefits and service management" (CO2). Here, it is a question of whether the health insurer wants to develop into a health advisor or stay with the core business. Therefore, use case prioritization should also follow strategic alignment considerations.

\section{Discussion}

Health insurers stand to benefit from AI adoption, as they typically have access to vast amounts of data. However, AI adoption is a challenging endeavor in practice. Extant research has thus far not investigated AI adoption specifically for health insurance. Therefore, we conducted explorative interviews with health insurance experts. We identified a plethora of AI use cases in the areas of predictive health, individualized service, anomaly detection, and operations enhance. In addition, we and found that health insurers are likely to prioritize these use cases according to implementation complexity and business orientation. In the following, we will discuss our findings against the background of extant research as well as our contributions to theory and practice, limitations and avenues for future research. 


\subsection{Implications for AI Adoption}

Previous research has identified various technological, organizational, and environmental factors that influence the decision of adopting AI, whereas AI has been mostly viewed as a monolithic concept. We explored how health insurers would use these factors to prioritize different use cases for AI adoption, finding that implementation complexity and business orientation are most likely the important factors. Our findings further suggest that health insurers, who in our sample had only little experience with AI, would focus on simpler use cases first to gather experience, whereas more complex use cases should be mastered later in the AI adoption.

Implementation complexity can mostly be described by use case implications, data accessibility, and data sensitivity. The importance of data in adequate quantity and quality has been repeatedly mentioned in literature as an organizational factor for AI adoption [10, $12,13]$. This is not surprising, as today's AI systems mostly learn from data. Besides, research has also acknowledged the role of environmental factors, such as the regulatory context and customer AI readiness [13, 17, 22]. These environmental factors encourage health insurers to prioritize use cases, as manifested by the factors use case implications and data sensitivity. However, we would have also expected to find that different types of AI technologies are an important factor for implementation complexity as well. For example, having a deep learning algorithm that cannot be precisely explained might raise implementation complexity [31]. Potentially, our interviewed experts were unaware of this potential factor because of AI adoption's early stages in health insurance.

As our study investigated prioritization criteria for different use cases (instead of adoption factors for $\mathbf{A I}$ as a monolithic technology), we conclude that not all factors are equally relevant for each AI use case. For example, data access or regulatory aspects must not be a challenge for AI use cases that focus on internal operations. As another example, the business value of predictive health might be more focused on patient health and cost reduction, whereas individualized services target customer satisfaction. Therefore, we conclude that research and practice should consider AI adoption in a more nuanced way to respect the nature and implications of different use cases and AI technologies.

Building on the initial research on AI adoption [e.g., 32], we contribute to the understanding of strategic considerations for AI adoption. First, we can see that certain AI use cases imply changes to the core business model of health insurers. For example, focusing on predictive health would position an insurer as a health advisor, whereas other use cases such as operations enhancement or anomaly detection target improvements of existing business processes. Hence, the prioritization of use cases goes alongside strategic considerations, highlighting the strategic value of AI. Second, we found that developing more complex use cases requires organizational commitment and investments in appropriate resources. For example, organizations first need to acquire new know-how or gather experiences with simpler use cases. Therefore, we conclude that $\mathrm{AI}$ adoption requires organizations to continuously adapt themselves (and their AI readiness) during the adoption process, as also proposed by Jöhnk, et al. [22]. This enables organizations to build AI capabilities and successfully approach more complex AI use cases. Third, AI adoption might stimulate organizations to involve new partners to form rich platform ecosystems [33] that fuel AI implementation. Such ecosystems could potentially consist of health insurers, doctors, hospitals, drug producers, and software companies [34]. Hence, there are new opportunities for collaboration and participation, which should be closely examined in the context of health insurance and AI adoption. The strategic relevance of $\mathrm{AI}$ also confirms the importance of industry and market characteristics as an environmental factor for $\mathrm{AI}$ adoption, as suggested by previous findings $[12,17]$.

\subsection{Contributions to Theory and Practice}

This study contributes to the ongoing discourse on the use of AI surrounding health care. We expand our understanding of AI adoption in health insurance with its unique regulatory context and data sensitivity. Specifically, we focus on the little explored area of AI use case prioritization. Thereby, we advance extant research on AI adoption that predominately investigated factor for the adoption decision early in the process. In addition, we provide a new perspective by differentiating between use cases (with different characteristics) rather than viewing $\mathrm{AI}$ as a monolithic concept. This revealed that previously known factors on AI adoption do not apply equally well for different use cases. We conclude that researchers and practitioners should take a more nuanced view of AI adoption to respect different use cases and AI technologies.

We also provide valuable insights for practice. Health insurance practitioners could use our findings as a starting point for their AI adoption decision making. We provide a structured list of AI use cases and criteria that can be used to prioritize use cases, enabling insurers with little AI experience to identify and prioritize suitable use cases and develop a roadmap for AI adoption. 


\subsection{Limitations and Future Research}

This study has certain limitations. To explore AI use cases in health insurance and identify respective prioritization criteria, we relied on a qualitative research approach. Although this approach is well aligned with our research questions, it is prone to inherent weaknesses. Among them is researcher bias when interpreting our interview data [26, 29]. Although it is impossible to completely erase researchers' personal views and preconceptions, we mitigated bias by considering multiple perspectives as part of our sampling strategy. Additionally, we focused on German statutory health insurance system in this study. Although we argue that our findings are well applicable to health insurers in other countries as well, our sample of health insurance representatives was limited to organizations that are beginning their AI adoption journey. Hence, more experienced organizations might consider different factors for use case prioritization, such as the complexity of different technologies. Finally, we conducted a total of seven interviews, as this study aimed at exploring AI use cases for health insurance and the respective prioritization criteria. Although we argue our data are sufficient and yielded interesting insights, we acknowledge that more data would further increase the confidence in our findings and potentially allow us to distill more generalizable theoretical contributions.

Our study opens up new avenues for future research. By conducting a longitudinal case study within a health insurance company, we gained additional insights into how use case prioritization might progressively shift as organizations gain further experience or change their strategy. In addition, future research might look into AI use case prioritization in other contexts regarding the healthcare domain. This would help to further refine and assess our findings, especially with regards to generalizability.

\section{Conclusion}

Health insurers can benefit from AI as they typically have access to vast amounts of data. However, practitioners struggle with AI adoption, and previous research lacks an overview of AI use cases in health insurance and prioritization criteria that can guide adoption. To address this, we conducted exploratory interviews in the German statutory health insurance system. We identified AI use cases in predictive health, individualized service, anomaly detection, and operation enhancement. We also found that health insurers are likely to prioritize these use cases by implementation complexity and business orientation. Experts recommend focusing on simple use cases first to gain experience with AI, before approaching more complex use cases that involve more strategic considerations.

We contribute to an ongoing discourse on AI adoption in healthcare research. Moreover, we provide a novel perspective on AI adoption by differentiating between use cases, instead of viewing AI as a monolithic concept. We conclude that research and practice should consider AI adoption in a more nuanced way. Our findings serve as a valuable tool for health insurance practitioners to support $\mathrm{AI}$ adoption decision making. We provide a structured list of AI use cases and criteria for use case prioritization.

\section{References}

[1] T. Davenport and R. Kalakota, "The potential for artificial intelligence in healthcare," Future healthcare journal, vol. 6, no. 2, pp. 94-98, 2019.

[2] P. Stone et al., ""Artificial Intelligence and Life in 2030." One Hundred Year Study on Artificial Intelligence," Stanford University, Stanford, USA, Report of the 20152016 Study Panel, 2016. Accessed: July 1, 2020. [Online]. Available: http://ai100.stanford.edu/2016-report

[3] J. He, S. L. Baxter, J. Xu, J. Xu, X. Zhou, and K. Zhang, "The practical implementation of artificial intelligence technologies in medicine," Nature medicine, vol. 25, no. 1, pp. 30-36, 2019.

[4] P. Hofmann, S. Oesterle, P. Rust, and N. Urbach, "Machine Learning Approaches along the Radiology Value Chain-Rethinking Value Propositions," presented at the 27th European Conference on Information Systems (ECIS), Stockholm \& Uppsala, Sweden, 2019.

[5] K. Klinker, M. Wiesche, and H. Krcmar, "Digital transformation in health care: Augmented reality for hands-free service innovation," Information Systems Frontiers, vol. 22, no. 6, pp. 1419-1431, 2020.

[6] M. Burkhart et al., "Sherlock in Health - How artificial intelligence may improve quality and efficiency, whilst reducing healthcare costs in Europe.," PricewaterhouseCoopers, 2017. [Online]. Available: https://store.pwc.de/de/pdf/2017/august/sherlock-inhealth-how-artificial-intelligence-may-improve-qualityand-efficiency-whilst-reducing-healthcare-costs-ineurope

[7] C. Auer, N. Hollenstein, and M. Reumann, "Künstliche Intelligenz im Gesundheitswesen [Artificial Intelligence in Healthcare]," in Gesundheit Digital [Digital Health], R. Haring Ed. Berlin: Springer, 2019.

[8] H. Benbya, T. H. Davenport, and S. Pachidi, "Artificial Intelligence in Organizations: Current State and Future Opportunities," MIS Quarterly Executive, vol. 19, no. 4, pp. ix-xxi, 2020.

[9] M. Tarafdar, C. M. Beath, and J. W. Ross, "Using AI to enhance business operations," Sloan Management Review, vol. 60, no. 4, pp. 37-44, 2019.

[10] T. Q. Sun and R. Medaglia, "Mapping the challenges of Artificial Intelligence in the public sector: 
Evidence from public healthcare," Government Information Quarterly, vol. 36, no. 2, pp. 368-383, 2019.

[11] T. Davenport and R. Ronanki, "Artificial intelligence for the real world," Harvard Business Review, vol. 96, no. 1, pp. 108-116, 2018.

[12] L. Pumplun, C. Tauchert, and M. Heidt, "A New Organizational Chassis for Artificial IntelligenceExploring Organizational Readiness Factors," presented at the 27th European Conference on Information Systems (ECIS), Stockholm \& Uppsala, Sweden, 2019.

[13] Q. Demlehner and S. Laumer, "Shall We Use It or Not? Explaining the Adoption of Artificial Intelligence for Car Manufacturing Purposes," presented at the 28th European Conference on Information Systems (ECIS), Virtual Conference, 2020.

[14] L. Kruse, N. Wunderlich, and R. Beck, "Artificial Intelligence for the Financial Services Industry: What Challenges Organizations to Succeed," presented at the 52nd Hawaii International Conference on System Sciences (HICSS), Hawaii, USA, 2019.

[15] M. Burkhart and S. Huesman-Koecke, "Das deutsche Gesundheitswesen auf dem Prüfstand [A Critical Assessment of German Healthcare]," PricewaterhouseCoopers, 2018. [Online]. Available: https://www.pwc.de/de/gesundheitswesen-undpharma/pwc-das-deutsche-gesundheitswesen-auf-dempruefstand.pdf

[16] D. Radić et al., "Digitalisierung im Krankenversicherungsmarkt [Digitalization in Health Insurance]," Fraunhofer IMW, 2018. [Online]. Available: https://www.imw.fraunhofer.de/content/dam/moez/de/do cuments/Studien/180928_Studie\%20IMW\%20Digitalisie rung\%20Krankenversicherungsmarkt.pdf

[17] K. Nam, C. S. Dutt, P. Chathoth, A. Daghfous, and M. S. Khan, "The adoption of artificial intelligence and robotics in the hotel industry: prospects and challenges," Electronic Markets, pp. 1-22, 2020.

[18] R. Depietro, E. Wiarda, and M. Fleischer, "The context for change: Organization, technology and environment," The processes of technological innovation, vol. 199, no. 0, pp. 151-175, 1990.

[19] E. M. Rogers, Diffusion of innovations, 5th ed. New York, USA: Free Press, 2003.

[20] H. J. Watson, "Preparing for the Cognitive Generation of Decision Support," MIS Quarterly Executive, vol. 16, no. 3, pp. 153-169, 2017.

[21] L. Baier, F. Jöhren, and S. Seebacher, "Challenges in the deployment and operation of machine learning in practice," presented at the 27th European Conference on Information Systems (ECIS), Stockholm \& Uppsala, Sweden, 2019.
[22] J. Jöhnk, M. Weißert, and K. Wyrtki, "Ready or Not, AI Comes-An Interview Study of Organizational AI Readiness Factors," Business \& Information Systems Engineering, vol. 63, no. 1, pp. 5-20, 2021.

[23] T. Fountaine, B. McCarthy, and T. Saleh, "Building the AI-powered organization," Harvard Business Review, vol. 97, no. 4, pp. 62-73, 2019.

[24] S. AlSheibani, C. Messom, and Y. Cheung, "Rethinking the Competitive Landscape of Artificial Intelligence," presented at the 53rd Hawaii International Conference on System Sciences (HICSS), Hawaii, USA, 2020.

[25] M. Garbuio and N. Lin, "Artificial intelligence as a growth engine for health care startups: emerging business models," California Management Review, vol. 61, no. 2, pp. 59-83, 2019.

[26] G. Walsham, "Doing interpretive research," European journal of information systems, vol. 15, no. 3, pp. 320-330, 2006.

[27] M. D. Myers and M. Newman, "The qualitative interview in IS research: Examining the craft," Information and organization, vol. 17, no. 1, pp. 2-26, 2007.

[28] H. K. Klein and M. D. Myers, "A set of principles for conducting and evaluating interpretive field studies in information systems," MIS quarterly, pp. 67-93, 1999.

[29] A. Strauss and J. Corbin, Basics of qualitative research. Sage publications, 1990.

[30] D. A. Gioia, K. G. Corley, and A. L. Hamilton, "Seeking qualitative rigor in inductive research: Notes on the Gioia methodology," Organizational Research Methods, vol. 16, no. 1, pp. 15-31, 2013.

[31] A. Asatiani, P. Malo, P. R. Nagbøl, E. Penttinen, T. Rinta-Kahila, and A. Salovaara, "Sociotechnical Envelopment of Artificial Intelligence: An Approach to Organizational Deployment of Inscrutable Artificial Intelligence Systems," Journal of the Association for Information Systems, vol. 22, no. 2, pp. 325-352, 2021.

[32] A. F. Borges, F. J. Laurindo, M. M. Spínola, R. F. Gonçalves, and C. A. Mattos, "The strategic use of artificial intelligence in the digital era: Systematic literature review and future research directions," International Journal of Information Management, vol. 57, pp. 1-16, 2020.

[33] A. Hein et al., "Digital platform ecosystems," Electronic Markets, vol. 30, no. 1, pp. 87-98, 2020.

[34] S. Hermes, T. Riasanow, E. K. Clemons, M. Böhm, and H. Krcmar, "The digital transformation of the healthcare industry: exploring the rise of emerging platform ecosystems and their influence on the role of patients," Business Research, vol. 13, no. 3, pp. 10331069, 2020. 